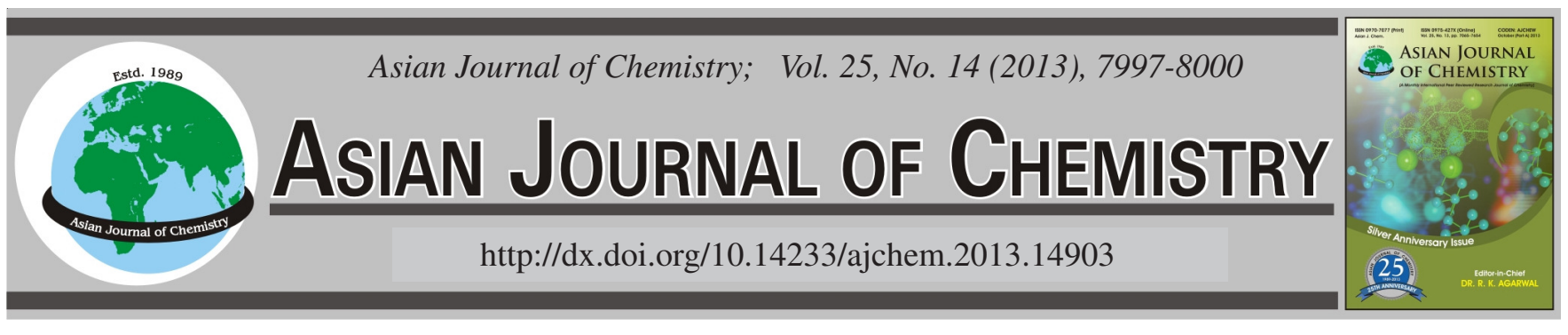

\title{
Study on the Influence of Modified Multi-Walled Carbon Nanotubes to the Shear Strength of Urea-Formaldehyde Resin Adhesive
}

\author{
Jintian Huang ${ }^{*}$, Kunling Tie and Yanfei Pan
}

College of Material Science and Art Design, Inner Mongolia Agricultural University, Hohhot, P.R. China

*Corresponding author: Fax: +86 471 4309408; Tel: +86 13947125748; E-mail: jintian_h@163.com

Multi-walled carbon nanotubes (MWCNTs) were modified successfully with concentrated sulfuric acid/nitric acid 3:1 (v/v) and anionic surfactant, then MWCNTs/urea-formaldehyde resin (UF) composites were prepared through mechanical mixing and ultrasonic method. The results show that the surface of MWCNTs was introduced carboxyl groups and the hydrophilic groups of $-\mathrm{OSO}_{3}{ }^{2-}$ after modification. Shear strength of MWCNTs/UF conposites increased by $90.7 \%$ comparing with pure UF. The optimal proportion of sodium dodecyl sulfate (SDS)/MWCNTs is 2:1 (wt. ratio), which the modified MWCNTs could be dispersive in UF resin, the mixing time decreased by adding surfactant.

Key Words: Multi-walled carbon nanotubes, Modified, Shear strength, Urea-formaldehyde.

\section{INTRODUCTION}

Due to their exceptional mechanical, thermal and electromagnetic properties, carbon nanotubes (CNTs) as a functional material have been using in different fields including nanoscale composites synthesis, polymer reinforcement materials ${ }^{1-4}$, electronic components, nano/bio-sensors, hydrogen containers ${ }^{5}$ and catalyst carrier. Because of abundant Van der Waals force, CNTs tend to agglomerate into bundles and difficult to reach homogeneous distribution in the polymeric matrix systems, it would be paid more attention on improving the dispersion. Surface modification of the CNTs is the main step before synthesis with other materials ${ }^{6,7}$. Some functional groups such as carboxyl and amino can be introduced on the surface of CNTs by using chemical treatment. Surfactants treatment can disentangle bundles of CNTs by the way of adsorption.

Urea-formaldehyde (UF) resin adhesive is a non-transparent thermosetting resin made from urea and formaldehyde heated in the presence of a mild base such as ammonia or pyridine. It is the major adhesive in the wood panel industrialization. However, due to the weak bonding strength, poor aging resistance, high formaldehyde emission and low water absorption. It is feasible to improve above properties by adding fiber reinforcements, thus the treated UF could be used in wide applications $^{8-10}$.

This paper investigated the inorganic nanotubes enhancing the shear strength of UF resin adhesive by mechanical mixing. The acid-treated MWCNTs was mixed with surfactants (SDS) to improve dispersibility in UF resin. We expect that the MWCNTs/UF composites would be used in wood processing with remarkable mechanical properties.

\section{EXPERIMENTAL}

Multi-walled carbon nanotubes (MWCNTs) were purchased from Shenzhen Nanotech. Port Co., Ltd. (Shenzhen, China), sodium dodecyl sulphate (SDS), sulfuric acid, nitric acid, urea, formaldehyde, ammonia aqueous solutions (10\%) of sodium hydroxide and aqueous solutions (10\%) of ammonium chloride were analytical grade. Poplar veneers were used as the plywood production.

KS-300EI type, ultrasonic cleaning device, Ningbo kesheng the ultrasonic equipment Co., Ltd. (Ningbo, China). Fourier transform infrared spectrometer (FT-IR), N EXUS type. the United States Thermo Nicolet company. scanning electron microscope (SEM), S-3400 N type. Hitachi, Japan.WDW-20A type microcomputer control electronic universal test machine, Jinan tianchen Co., Ltd (Jinan, China).

Treatment of MWCNTs: MWCNTs is easy to aggregate into bundles due to high surface energy. Acidic functional groups can be introduced on the MWCNTs surface by acid treatment can produce acidic. MWCNTs exhibit chemical stability after modification. The MWCNTs were first treated with a $3: 1(\mathrm{v} / \mathrm{v})$ mixture of concentrated sulfuric acid and nitric acid assisting with ultrasonication for $4 \mathrm{~h}$ in room temperature, purposing to enhance MWCNTs surface functionalization and remove impurities, such as amorphous carbons and catalyst 
particles ${ }^{11}$. Then, the oxidized carbon was filtrated and washed with distilled water until the $\mathrm{pH}$ value closing to neutral, then dried in vacuum oven at $80{ }^{\circ} \mathrm{C}$ for $12 \mathrm{~h}$.

Prepared the $0.1 \%$ concentration solutions of surfactant (SDS) in deionized (DI) water, then acid-treated MWCNTs were added into above surfactant. The SDS/MWCNTs ratio (wt.) adjusted to 1:5. The mixtures were sonicated for $0.5 \mathrm{~h}$; the final modified MWCNTs were obtained after filtration.

Preparation of urea-formaldehyde resins: Urea-formaldehyde resin was prepared following traditional alkalineacid two-step reaction. The formaldehyde was adjusted to $\mathrm{pH}$ 8.5-9.0 with aqueous $\mathrm{NaOH}, \mathrm{A}$ quarter of the total amount of urea was added, heating to $60{ }^{\circ} \mathrm{C}$ under reflux, adding the second urea (a quarter of the total), continuing to heat until at $80{ }^{\circ} \mathrm{C}$, then the rest of urea was added to complete the methylolation reaction for $0.5 \mathrm{~h}$. The condensation reaction in condition of adding aqueous $\mathrm{NH}_{4} \mathrm{Cl}$ adjusting to the $\mathrm{pH} 4.8$ and the reactant were carried out until it appeared emulsion phenomenon in water with a temperature of $40{ }^{\circ} \mathrm{C}$. The UF resin was cooled immediately, following by adjusting the $\mathrm{pH}$ value in the range of 8.5-9.0 using ammonia, the UF resin gained finally. The mole ratio between formaldehyde and urea is 1.93 .

Preparation of MWCNTs/UF: The functionalized MWCNTs and surfactant were added into the UF adhesive with different ratios. The mixture distributed uniformaly after adding $1 \%$ of $\mathrm{NH}_{4} \mathrm{Cl}$ as curing agent. The MWCNTs/UF composites was used as an adhesive for poplar veneers enhancing the mechanical property, before that the poplar veneers was treated by polishing. The samples were extruded (1.0 MPa) and curled at room temperature for $24 \mathrm{~h}$ to measure the shear strength according to GJB1709-1993 (Fig. 1). Specimen size: $100 \mathrm{~mm} \times 25 \mathrm{~mm} \times 6 \mathrm{~mm}$, sizing area: $12.5 \mathrm{~mm} \times 25 \mathrm{~mm}$.

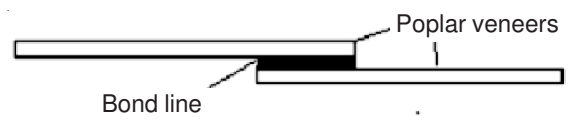

Fig. 1. Shear strength measurement

\section{RESULTS AND DISCUSSION}

FT-IR analysis of MWCNTs: Fig. 2 shows the FT-IR spectra of pristine MWCNTs (a) and after acid-treated modification (b). An absorbance peak was detected at $1714 \mathrm{~cm}^{-1}$ corresponding to the $\mathrm{C}=\mathrm{O}$ stretching, the peaks at $3400 \mathrm{~cm}^{-1}$ nearby and $1201 \mathrm{~cm}^{-1}$ indicating vibrational bond of $-\mathrm{OH}$ and deformation of C-O groups. The appearance of these groups provides evidence that carboxyl groups have been produced on MWCNTs surface. Due to $\mathrm{HNO}_{3}$ decompose free oxygen atoms, which oxidize the CNTs at defections and cap ends. The other peaks at $2300 \mathrm{~cm}^{-1}$ nearby, $2895 \mathrm{~cm}^{-1}$ and 1604 (a) 1562 (b) $\mathrm{cm}^{-1}$ can be assigned to water interference, asymmetric and symmetric stretching of $-\mathrm{CH}_{2}$, stretching vibrations of $\mathrm{C}-\mathrm{C}$ bonds, respectively.

It indicated that the peaks at 2918, 2854 and $1469 \mathrm{~cm}^{-1}$ disappeared for SDS-MWCNTs (the SDS/MWCNTs ratio is 2:1) comparing with pure SDS from Fig. 3 which are $-\mathrm{CH}$ and $-\mathrm{CH}_{3}$ group, the strong peak at $1219 \mathrm{~cm}^{-1}$ remained corresponding to stretching vibration of $-\mathrm{OSO}_{3}{ }^{2-}$ (alkyl sulfate). The result shows that the hydrophilic groups of $-\mathrm{OSO}_{3}{ }^{2-}$ was introduced

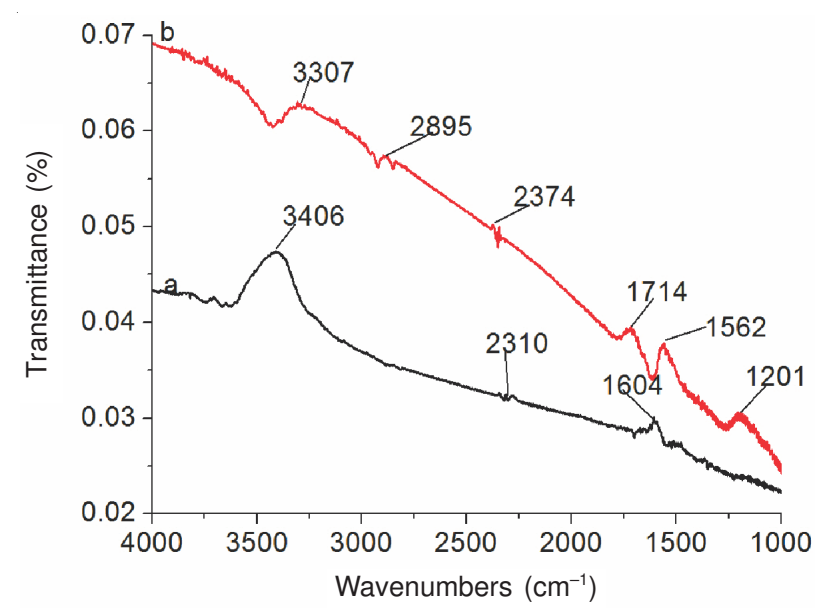

Fig. 2. FT-IR spectra of pristine MWCNTs (a)

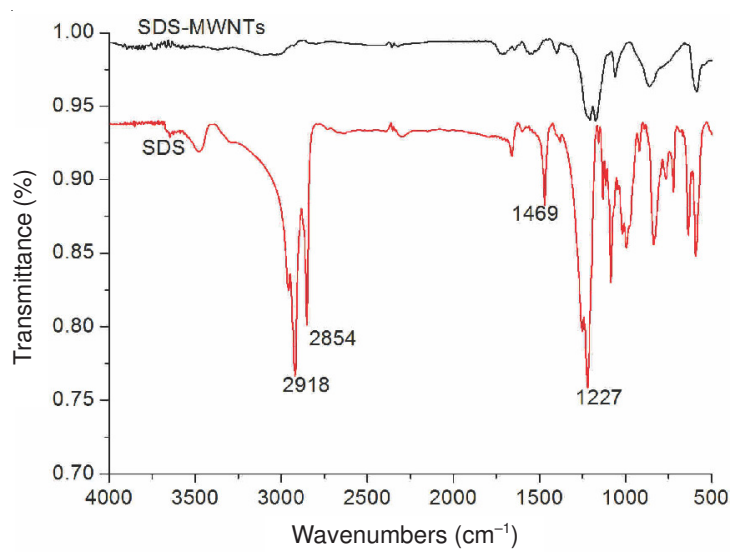

Fig. 3. FT-IR spectra of SDS-MWCNTs, SDS acid-treated MWCNTs (b)

on surface of MWCNTs which plays a role for the dispersion of MWCNTs.

SEM analysis of MWCNTs: The SEM was investigated to characterize the morphologies of pristine MWCNTs and acid-treated MWCNTs. The crosslinked and entangled MWCNTs was clearly observed from Fig. 4a. It shows that pristine MWCNTs is disorderly and unsystematic. The structure of MWCNTs was not destroyed after acid treatment (Fig. $4 \mathrm{~b}$ ), which relative homogeneous, although some defects and aggregation are still observed.
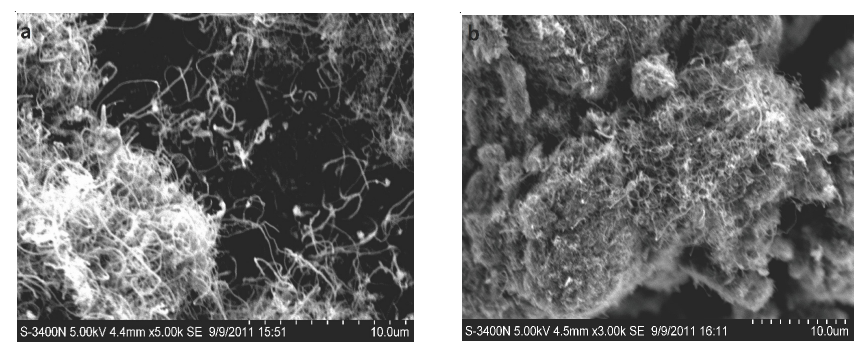

Fig. 4. SEM images of pristine MWCNTs (a), acid-treated MWCNTs (b)

\section{Mechanical properties analysis}

Shear strength results: Table-1 shows the shear strength of MWCNTs/UF composites indicating that the results in an upward at first and then downward in different ultrasonic time, the same trend is suitable for different content of MWCNTs and different SDS/MWCNTs ratio. 
TABLE-1

VALUES OF SHEAR STRENGTH

\begin{tabular}{cccccc}
\hline & Variable & $\begin{array}{c}\text { Min } \\
(\mathrm{MPa})\end{array}$ & $\begin{array}{c}\text { Max } \\
(\mathrm{MPa})\end{array}$ & $\begin{array}{c}\text { Average } \\
(\mathrm{MPa})\end{array}$ & $\begin{array}{c}\text { Standard } \\
\text { deviation }\end{array}$ \\
\hline & 0 & 1.511 & 2.013 & 1.8222 & 0.2294 \\
Ultrasonic time & 2 & 1.788 & 3.183 & 2.5642 & 0.5641 \\
(h) & 4 & 2.295 & 3.696 & 2.879 & 0.5453 \\
& 6 & 2.276 & 3.029 & 2.6532 & 0.3229 \\
& 8 & 1.439 & 2.686 & 2.116 & 0.4496 \\
\hline & 0 & 1.142 & 1.926 & 1.4994 & 0.3802 \\
Content of & 0.1 & 1.788 & 3.183 & 2.5642 & 0.5651 \\
MWCNTs (\%) & 0.3 & 2.304 & 2.965 & 2.6294 & 0.3078 \\
& 0.5 & 2.128 & 3.240 & 2.8588 & 0.4492 \\
& 0.7 & 2.138 & 3.103 & 2.7104 & 0.4049 \\
\hline \multirow{5}{*}{ SDS/MWCNTs } & 0 & 1.511 & 2.013 & 1.8222 & 0.2294 \\
ratio & 1 & 0.7083 & 2.792 & 1.80526 & 0.7858 \\
& 2 & 2.122 & 3.115 & 2.5576 & 0.3883 \\
& 3 & 2.122 & 3.115 & 2.5576 & 0.3883 \\
& 4 & 1.256 & 2.369 & 1.7698 & 0.4035 \\
& 4 & 0.2788 & 1.946 & 1.2033 & 0.6213 \\
\hline
\end{tabular}

The interaction between these values has been examined with variance analysis ${ }^{8}$ (Table-2). It shows that the effect of different content of MWCNTs, ultrasonic time and SDS/ MWCNTs ratio on the shear strength has been investigated significance with 0.0001 margin of error, significance with 0.003 margin of error and notable with 0.0122 margin of error, respectively.

Shear strength of MWCNTs/UF adhesion with different ultrasonic time: For different ultrasonic time (sonication in water bath), the content of MWCNTs as a constant $(0.1 \%)$ was setted. It is seen that the shear strength increased $c a .33 .5 \%$ at $2 \mathrm{~h}$, which is the largest rate. The shear strength achieves maximum values at ultrasonic time of $4 \mathrm{~h}$ (equivalent to $0.5 \%$ MWCNTs in UF), then decrease slowly next $4 \mathrm{~h}$, it was possibly due to the ultrasonic cavitation ${ }^{12}$. The generation of shock waves and micro-jet due to the bubbles in media formation and collapse instantly, which significantly favour to the even distribution of MWCNTs in UF resin. After a period of ultrasonic time, the mixture tends to be uniform and stable suspension. It can improve the shear strength taking advantage of MWCNTs mechanical properties. However, with the extension of ultrasonic time, it increases the probability of collisions between particles, resulting in the aggregation of MWCNTs, which causes the decrement of shear strength.

Shear strength of MWCNTs/UF adhesion with different concentration of MWCNTs: For different concentration of MWCNTs, we set ultrasonic time as a constant $(2 \mathrm{~h})$. It is seen that the shear strength achieve maximum values at a MWCNTs loading of $0.5 \%$, whereas the shear strength increased to more than $90 \%$ compared with the pure UF. The values changed slightly after adding MWCNTs between 0.5 and $0.7 \%$. The possibe reason could be explained that the functional MWCNTs caused crosslinking with active groups in UF resin, which improved the cohesion of UF. However, with the MWCNTs increasing, the aggregation of MWCNTs ${ }^{13}$ occured due to surface energy. Leading to the mechanical properties decreased in a relatively short dispersed time.

Shear strength of MWCNTs/UF adhesion with different ratio of SDS/MWCNTs: SDS has been used as a dispersant for MWCNTs with good results. In the experiment, it is observed that the viscosity of UF resin increased rapidly by using SDS. The mixture obtained in condition of stirring uniformly. When the SDS/MWCNTs ratio was up to 2:1, which was a turning point, the shear strength achieved maximum value. The interaction between SDS and MWCNTs are due to hydrophobic interactions of surfactants and steric hindrance causing by electrostatic repulsive forces of MWCNTs ${ }^{14}$. The ratio of SDS/ MWCNTs has significant effect on dispersion. The appropriate ratio could balance the competition between the adsorption and desorption of SDS on MWCNTs, in this case, the SDS would adsorb on the surface of MWCNTs forming micelles to ensure a homogeneous distribution. The lower ratios lead to poor dispersion, while the higher ratios result to presence of the excess surfactant as the second phase, which the shear strength can be reduced by above two aspects.

Dispersion of MWCNTs in UF resin: The dispersion of MWCNTs in UF resin was examined with the composite fracture surfaces as shown in Fig. 5(a-c), the concentration of MWCNTs was up to $0.5 \%$. It shows that the white points in figures represent the broken MWCNTs, which the interfacial interaction occurred between MWCNTs and UF. From Fig. 5a, a number of pristine MWCNTs are found entangling together and distributing randomly. There are acid-treated MWCNTs pulled out from the matrix surface from Fig. 5b, which demonstrate the interfacial bonding decreased. The distribution uniformly from Fig. 5c, SDS assisting acid-treated MWCNTs shows good dispersibility, but still the entangled MWCNTs wrapped by UF resin, reducing mechanical performance of composites $^{15}$. It indicated that the MWCNTs dispersed in UF resin in uneven system from Fig. 5, which is the further issue to be solved in future.

TABLE-2

SHEAR STRENGTH VARIANCE ANALYSIS RESULTS

\begin{tabular}{clccccc}
\hline & Variance source & DF & Sum of squares & Mean square & F Value & Level of significance \\
\hline \multirow{2}{*}{ Content } & Inter groups & 4 & 7.2359 & 1.8090 & 9.78 & 0.0001 \\
MWCNTs & Within groups & 20 & 3.6980 & 0.1849 & - & - \\
& Total & 24 & 10.9339 & - & - & - \\
\hline \multirow{3}{*}{ Ultrasonic time } & Inter groups & 4 & 3.5347 & 0.8837 & 5.74 & 0.0030 \\
& Within groups & 20 & 3.0813 & 0.1541 & - & - \\
& Total & 24 & 6.616 & - & - & - \\
\hline \multirow{2}{*}{ SDS/MWCNTs } & Inter groups & 4 & 4.6318 & 1.1579 & 4.23 & - \\
ratio & Within groups & 20 & 5.4791 & 0.2739 & - & - \\
& Total & 24 & 10.1109 & - & - & -0122 \\
\hline
\end{tabular}

Note: $\mathrm{F}_{0.1}(4,20)=2.25, \mathrm{~F}_{0.05}(4,20)=2.87, \mathrm{~F}_{0.01}(4,20)=4.43$. 


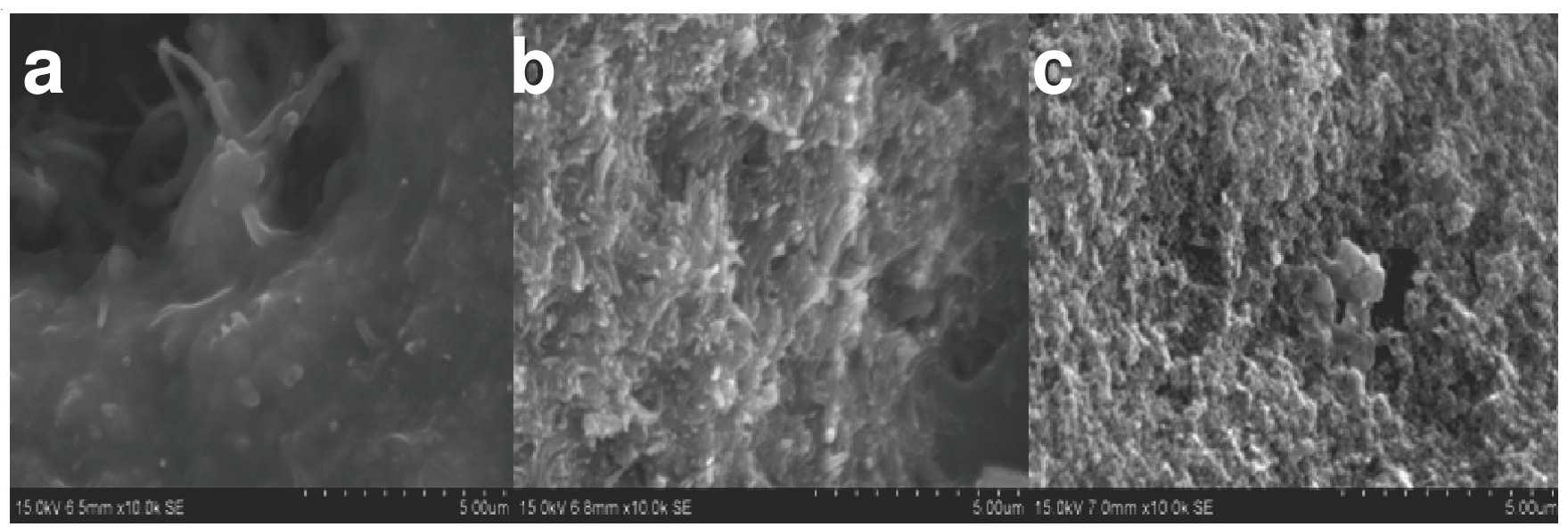

Fig. 5. SEM images of pristine MWCNTs/UF (a), acid-treated MWCNTs/UF (b), SDS modified MWCNTs/UF (c)

\section{Conclusion}

The carboxylic functional groups were introduced on the surface of MWCNTs by acid treatment, confirming by FT-IR spectra and SEM analysis. The MWCNTs/UF resin compsites could enhance the shear strength significantly. Appropriate ultrasound time contributes to the dispersion of MWCNTs in UF matrix. Surfactant can reduce the mixing time but to choose the proper amount.

The study on nano-modified UF resin is still in the initial stages further researches on the dispersion of MWCNTs in UF adhesives and fabrication of MWCNTs/UF composites should be analyzed.

\section{ACKNOWLEDGEMENTS}

This work was sponsored by the National Forestry Bureau 948 Project of China (2011-4-07) and Inner Mongolia Natural Science Fund Major Projects of China (2011ZD07).

\section{REFERENCES}

1. S. Ijima, Nature, 354, 56 (1991).

2. N.H. Tai, M.K. Yeh and J.H. Liu, Carbon, 42, 2774 (2004).
3. T. Ogasawara, Y. Ishida, T. Ishikawa and R. Yokota, Composites A, 35, 67 (2004).

4. F.H. Gojny, M.H.G. Wichmann, U. Köpke, B. Fiedler and K. Schulte, Comp. Sci. Technol., 64, 2363 (2004).

5. C. Liu, Y.Y. Fan, M. Liu, H.T. Cong, H.M. Cheng and M.S. Dresselhaus, Science, 286, 1127 (1999).

6. M.D. Clark, S. Subramanian and R. Krishnamoorti, J. Colloid Interf. Sci., 354, 144 (2011).

7. Linda Vaisman, H. Daniel Wagner and Gad Marom, Adv. Colloid Interf. Sci., 128-130, 37 (2006).

8. C. Sensogut, M. Ozalp and H. Yesil, Int. J. Adhes. Adhes., 29, 589 (2009).

9. G.A. Shen, S. Namilae, N. Chandra, Mater. Sci. Eng. A, 429, 66 (2006).

10. S. Kim and H.-J. Kim, Int. J. Adhes. Adhes., 25, 456 (2005).

11. G.H. Gao and S.G. Chen, Adv. Mater. Res., 79-82, 609 (2009).

12. G.C. $\mathrm{Xu}$ and L.D. Zhang, Chemistry Industry Press: Nanocomposite, pp. 1-24.115-132 (2002).

13. J. Rausch, R.-C. Zhuang and E. Mader, Composites A, 41, 1038 (2010).

14. S.J. Park, H.J. Jeong and C.W.A. Nah, Mater. Sci. Eng. A, 385, 13 (2004).

15. L.Q. Liu and H.D. Wagner, Comp. Sci. Technol., 65, 1861 (2005). 